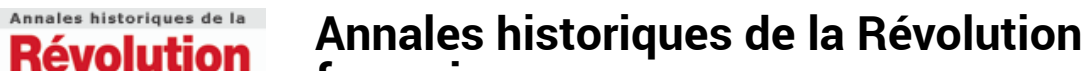

française française

367 | janvier-mars 2012

Théâtre et révolutions

\section{Gilles CANDELA, L'armée d'Italie (1792-1797). Des missionnaires armés à la naissance de la guerre napoléonienne}

\section{Annie Crépin}

\section{OpenEdition}

Édition électronique

URL : https://journals.openedition.org/ahrf/12391

DOI : 10.4000/ahrf.12391

ISSN : 1952-403X

Éditeur :

Armand Colin, Société des études robespierristes

\section{Édition imprimée}

Date de publication : 1 mars 2012

Pagination : 233-236

ISSN : 0003-4436

\section{Référence électronique}

Annie Crépin, «Gilles candeLA, L'armée d'Italie (1792-1797). Des missionnaires armés à la naissance de la guerre napoléonienne », Annales historiques de la Révolution française [En ligne], 367 | janvier-mars 2012 mis en ligne le 12 septembre 2012, consulté le 01 juillet 2021. URL : http://journals.openedition.org/ ahrf/12391; DOI : https://doi.org/10.4000/ahrf.12391

Ce document a été généré automatiquement le 1 juillet 2021

Tous droits réservés 


\title{
Gilles CANDELA, L'armée d'Italie (1792-1797). Des missionnaires armés à la naissance de la guerre napoléonienne
}

\author{
Annie Crépin
}

\section{RÉFÉRENCE}

Gilles CANDELA, L'armée d'Italie (1792-1797). Des missionnaires armés à la naissance de la guerre napoléonienne, préface de Francis Pomponi, Rennes, Presses universitaires de Rennes, 2011, 402 p., ISBN 978-2-7535-1284-9, $22 €$.

1 Gilles Candela a soutenu avec succès une thèse que ce livre rend enfin accessible à un large public d'historiens et historiennes et pas seulement à ceux et celles qui se consacrent à l'étude du fait militaire. Il s'agit d'une somme de plus de 400 pages, qui se place résolument dans le champ de la nouvelle histoire militaire et même dans celui de la nouvelle histoire bataille. L'ouvrage est fondé sur une analyse à la fois culturelle, sociale et politique du combat et aussi de la campagne militaire, et se réfère de façon bien venue (mais sans céder à l'effet de mode) au débat actuel à propos de la guerre «totale» ou de la guerre «absolue». Gilles Candela réussit la gageure de porter un regard neuf sur des sujets qui semblaient « rebattus » et qui étaient même devenus des mythes: la campagne d'Italie de 1796-1797 et Bonaparte au début de sa carrière. L'originalité de l'œuvre consiste précisément en ce que les deux premières parties sont consacrées à l'armée d'Italie avant Bonaparte. Ce déplacement d'accent permet ainsi de « revisiter » la légende napoléonienne.

2 L'ouvrage est bien écrit dans un style clair et est structuré selon un plan classique de type chronologique. La première partie intitulée «La naissance d'une armée républicaine " s'attache à analyser la gestation d'une armée nouvelle. Elle s'ouvre sur l'étude prosopographique de la composition sociale du corps des officiers et de son évolution que l'auteur juge particulièrement révélatrice de la mutation de l'instrument 
de guerre au début des guerres de la Révolution. Cette analyse est mise en lien étroit avec les origines intellectuelles de ces officiers, qui ne manquent pas d'avoir des conséquences sur la conception de la guerre à mener. Mais, dit Gilles Candela dans son introduction, cette machine de guerre fut aussi le résultat d'un État en révolution (p. 18), autrement dit de l'action des représentants en mission. Il y a d'excellentes pages sur les liens entre pouvoir politique, que ceux-ci incarnent de façon magistrale dans le cas étudié ici, et pouvoir militaire. Ces liens, très forts jusqu'à l'automne 1794, ont un retentissement majeur sur la conduite de la guerre. C'est grâce aux représentants en mission dont certains furent des personnalités majeures, Augustin Robespierre au premier chef, Ricord qui bénéficia du charisme du premier, mais aussi Barras, que s'établirent une unité de direction et une coordination de toutes les forces combattantes du Sud-Est, l'armée des Alpes, l'armée d'Italie et les divisions affectées au siège de Toulon. Les représentants en mission ne se mêlaient pas du détail des manœuvres mais imprimaient l'impulsion stratégique et politique (p. 67). Ils surent aussi agir avec pragmatisme et discernement: ils évitèrent les nominations ou les promotions de complaisance et formèrent une méritocratie d'officiers qui étaient à la fois révolutionnaires convaincus et des militaires compétents.

3 Ils surent enfin, tout comme leurs collègues auprès des autres armées, instaurer une nouvelle discipline propre à des hommes libres devenus citoyens vertueux, voire missionnaires armés. L'auteur souligne, également dans son introduction, que, tandis qu'en 1793, il n'existait ni armée d'Espagne ni armée d'Allemagne, l'armée d'Italie avait quelque chose de messianique du fait de son nom, suggérant que cette armée ne se battait pas pour une frontière naturelle mais pour exporter les valeurs de la Révolution. L'armée d'Italie eut même une réputation jacobine, en réalité seule une minorité était politisée. Initialement ces «missionnaires armés » ne se privèrent pas de déserter ni d'exercer toutes sortes de violences à l'encontre des populations et de leurs camarades, qui contrevenaient singulièrement à leur aura de patriotes. Peu à peu, la désertion fut combattue et la violence fut canalisée. Les représentants en mission instaurèrent une justice très codifiée qui eut un caractère expérimental (p. 109) et fait de l'armée d'Italie, sur ce point aussi, un laboratoire.

4 Mais l'action des officiers supérieurs fut elle aussi décisive, la justice devint un instrument disciplinaire entre leurs mains et transforma - pour le meilleur comme pour le pire - les citoyens soldats en militaires dociles même si cette docilité envers les chefs - qui apparaît donc avant que Bonaparte ne soit placé à la tête de l'armée d'Italie - ne fut pas le retour à une discipline d'Ancien Régime.

5 La seconde partie "Un laboratoire de la guerre nouvelle " contient des chapitres éclairants et parmi les meilleures pages du livre. On citera par exemple l'analyse de la guerre subversive, on dirait aujourd'hui idéologique, celle du maniement de la propagande et, en corrélation, l'étude de «l'utilisation» des patriotes italiens, des fuorusciti, dans ce type de guerre, grâce notamment à une presse qui émane d'euxmêmes et pas seulement des Français ; Gilles Candela montre d'ailleurs que c'est dans ce contexte et donc avant Bonaparte que naquit le mythe de l'armée d'Italie. En outre, grâce à ce point, l'ouvrage ne se cantonne pas au "franco-français » et constitue un apport à l'histoire de l'Italie et à la genèse de son unité, ainsi qu'aux rapports complexes " occupés-occupants » ou " libérateurs-libérés », devenus un thème majeur de la nouvelle histoire militaire. On citera aussi parmi les points forts de cette seconde partie la présentation des différents types de guerre ainsi que les raisons politiques et 
culturelles - et pas seulement militaires - du choix de tel ou tel type, enfin le rôle des représentants en mission dans ces choix. L'auteur a le grand mérite à ce propos de rappeler la différence entre guerilla et petite guerre trop souvent confondues, alors que la première est menée contre les barbets contre lesquels les Français retournent leurs propres méthodes, la seconde est une guerre de positions, une guerre de montagne, totalement différente également de la guerre réglée menée par les armées du nord et de l'est, une guerre qui fait la part belle aux petits groupes mobiles, combattant en tirailleurs et prenant l'initiative, même lorsqu'ils sont simples soldats. Cette expérience devait s'avérer décisive lors de la campagne ultérieure de Bonaparte.

On citera enfin l'étude de l'effort de guerre, dans ses dimensions économique et logistique certes, avec l'accent mis sur la «bataille du ravitaillement » et un regard neuf sur les services de renseignement, mais aussi dans sa dimension humaine, avec de très bonnes pages concernant les rapports entre les financiers et les représentants en mission désireux d'assurer à tout prix l'approvisionnement des troupes ainsi qu'à propos des services de santé et des hôpitaux. On retrouvera ces sujets dans la dernière partie avec l'éclairage suggestif qui leur est donné.

7 En tout cas, l'auteur met à mal la légende d'une armée d'Italie affamée et démunie avant l'arrivée de Bonaparte. De même c'est avant celle-ci et grâce aux réformes de Schérer, en particulier un nouvel amalgame, que seule la quintessence des combattants demeure dans l'armée. Celle-ci à la tête de laquelle se trouve un état-major renouvelé est capable désormais de s'adapter - relativement - à de nouvelles tactiques et à une forme de guerre plus manœuvrière.

D'où le titre de la troisième partie « Mythe et réalité de la campagne d'Italie ». Gilles Candela déconstruit l'image d'un Bonaparte deus ex-machina. Il analyse ici minutieusement chacune des batailles de la campagne de 1796-1797 en montrant comment Bonaparte s'appuie sur l'expérience antérieure de ses soldats. "On ne peut envisager la campagne d'Italie sans l'utilisation généralisée des techniques de la petite guerre »

(p. 251). Mais, même s'il tente à Castiglione - saut qualitatif - de les faire manœuvrer en vue d'une bataille rangée, il se heurte aussi aux limites de ces combattants. Seule l'arrivée des unités de l'armée du Rhin et de Sambre-et-Meuse accélère un apprentissage qui démontre tous ses mérites à la bataille du Tagliamento: pour la première fois, l'ordre mixte qui devait être la formation classique des troupes françaises du Consulat et de l'Empire fait son apparition sur un champ de bataille. Au risque de "dénaturer " l'armée d'Italie avec l'adjonction d'hommes plus expérimentés mais n'ayant pas l'état d'esprit des soldats qui la composaient à l'origine. Il se produit donc le même processus que dans le domaine de la justice et de la discipline.

9 Cette dernière partie montre aussi l'instrumentalisation de la guerre idéologique et la subordination des perspectives politiques aux impératifs stratégiques et à ceux d'une vaste entreprise économique qui renfloue les caisses du Directoire. Bonaparte ne croit plus à la guerre révolutionnaire. Certes, il joue de main de maître de la réputation jacobine de l'armée d'Italie mais c'est soit à des fins de politique intérieure soit pour accentuer la pression sur les états adversaires de la France. Vis-à-vis des patriotes italiens en attente de liberté et vis-à-vis des populations, c'est tout autre chose, au risque sur ce point aussi d'abandonner les idéaux de l'an II. La campagne d'Italie marque la transformation progressive d'une armée de «missionnaires armés » en une armée de maintien de l'ordre, même si celui-ci ne se réduit pas à une pure et simple 
répression et même si beaucoup d'officiers souhaitent sincèrement la régénération de la nation italienne.

10 Cet ouvrage très dense peut devenir une référence pour les étudiants qui s'engagent dans la voie de la recherche, car, lorsque l'auteur évoque l'armée d'Italie comme « le » laboratoire de la guerre contemporaine, il faut se demander si les autres armées de la Révolution ne l'ont pas été elles aussi d'une façon différente. 